\title{
EFECTO DE LA TEMPERATURA Y TIEMPO DE SECADO EN LA EXTRACCIÓN DE GRASA O MANTECA DE SEMILLAS (GRANOS O ALMENDRAS) DE CACAO (Theobroma cacao) DE LA VEREDA TOTARE, MUNICIPIO DE ALVARADO, DEPARTAMENTO DEL TOLIMA
}

\author{
Diego Alberto Marín Idárraga ${ }^{19}$
}

\begin{abstract}
RESUMEN
La extracción de grasa en el cacao fue estudiada en granos o almendras de Teobroma cacao de la variedad criolla para diferentes temperaturas $\left(45^{\circ} \mathrm{C}\right.$ y ambiente $28^{\circ} \mathrm{C}$ promedio $)$ y tiempos 12 y $24 \mathrm{~h}$.
\end{abstract}

Los granos o almendras fueron colocados en una fibra para su fermentación, ya que este es el procedimiento que realizan las comunidades indígenas, los campesinos y algunos cacaoteros de la región, de tal forma que el cacao se comercializa fermentado, o en proceso de fermentación. La función principal de la fermentación en fibras es quitar la pulpa que las rodea; este proceso se realizó durante seis días y luego se procedió a un secado. Las experiencias se realizaron con cuatro muestras a temperaturas y tiempos diferentes. El contenido de grasa se determinó utilizando un método de lixiviación con ayuda del solvente Éter-etílico.

De acuerdo con los resultados de este estudio, los granos de cacao se vieron afectados por las dos variables a analizar: tiempo y temperatura, concluyendo que el método en que mayor porcentaje de grasa se obtuvo fue el que se sometió a una temperatura de $45^{\circ} \mathrm{C} \mathrm{y}$ un tiempo de $24 \mathrm{~h}$. El estudio busca a su vez que se pueda realizar experimentalmente en variedades híbridas y en cacaos especiales del departamento del Tolima y que sea base para investigaciones futuras.

Palabras clave: determinación, fermentación, granos, lixiviación, secado.

\begin{abstract}
The extraction of fat in cocoa was studied in grains or almond of "Teobroma cacao" native variety for different temperatures $\left(45^{\circ} \mathrm{C}\right.$ and ambient $28^{\circ} \mathrm{C}$ average) and times (12 and $24 \mathrm{~h}$ ).

The grains or almonds were placed in a fiber for fermentation, since this is the procedure carried out by indigenous communities and farmers and some cocoa in the region, so that

19 Tutor ECBTI, del CEAD Ibagué, ZONA SUR; laboratorio de Bromatología del hospital Federico Lleras Acosta Correo electrónico: diegomarin33@hotmail.com. Nombre del Grupo de Investigación: GIEPRONAL, Nombre del Semillero: Biotecal, Red de Investigación: Producción Sostenible, Visibilidad y Gestión de Conocimiento, Escuela: Ciencias básicas, tecnologías e ingenierías ECBTI.
\end{abstract}


the fermented cocoa markets or in the process of fermentation, the main function of the fermentation of fiber is to remove the pulp that surrounds them, a process was done during six days and then it was proceeded to drying. The experiments were performed with four samples at different temperatures and times. The fat content was determined using a leaching method using solvent-ethyl ether.

According to the results of this study, the cocoa beans were affected by the two variables to analyze time and temperature, concluding that the method in which a higher percentage of fat was obtained which was subjected to a temperature of $45^{\circ} \mathrm{C}$ and a time of $24 \mathrm{~h}$. The study seeks to turn that can be done experimentally in hybrid varieties and special cocoa department of Tolima, by the same method for future research.

Key words: identification, fermentation, grains, leaching, drying.

\section{INTRODUCCIÓN}

Las grasas y aceites constituyen hoy, junto con las proteínas y carbohidratos y vitaminas, uno de los cuatro componentes más importantes de los alimentos consumidos por hombres y animales. Estos proporcionan energía, sabor, olor y propiedades lubricantes a los alimentos preparados. Los alimentos en forma de helados, mayonesa, margarinas, chocolate, revestimiento de confitería, adornos de pastelería, quesos y otros productos mantienen sus cualidades por la presencia de las grasas que tienen una alta adhesión y consistencia prolongada. Las grasas y aceites comestibles tienen su origen en los tejidos de los animales y plantas. Una definición sencilla de las grasas es que son materiales oleaginosos que están en estado sólido, la grasa a extraer específicamente es la que se encuentra presente en las almendras o granos de Cacao. El departamento del Tolima es uno de los principales productores de Cacao de Colombia dada la importancia cultural e histórica de este grano en las poblaciones indígenas y la proyección agrícola del departamento.

La manteca de cacao según la definición del Comité de Codex Alimentarium en productos de chocolatería "es la grasa producida de una o más de las siguientes fuentes: granos de cacao, licor de cacao (masa de cacao), torta de cacao y aquella extraída mediante procesos mecánicos y/o por la vía de solventes permitidos, de la torta o polvo de cacao fino".

La grasa o manteca de cacao es utilizada en la industria de la confitería en general y en la fabricación de barras de chocolate y energéticas, en particular. La razón fundamental por la que es utilizada se debe a su incomparable punto de fusión, a temperatura por debajo de 26 ${ }^{\circ} \mathrm{C}$, es dura y brillante, funde rápidamente y por completo a la temperatura corporal (Riendo, 1998). Además, posee un delicioso y característico olor a chocolate, benéfico para la industria chocolatera, que realza sus cualidades organolépticas y sensoriales.

Por lo tanto, en este estudio solamente se realizarán observaciones, muestreo al azar, diseño y análisis experimental, operaciones unitarias practicadas en laboratorio, tabulación, 
sistematización y confrontación de resultados esperados respecto de los obtenidos en granos de cacao de la variedad criolla o nativa del municipio de Alvarado.

\section{OBJETIVO GENERAL}

Determinar el efecto de la temperatura y tiempo de secado, en el porcentaje de grasa o manteca que se puede extraer.

\section{OBJETIVO GENERAL}

Confrontar estos tiempos y temperaturas y observar eficiencias en la extracción, y en los niveles óptimos de manteca (como producto final) en proporción cuantitativa.

Determinar tiempos y temperaturas recomendados, y a su vez, en una segunda fase del experimento, confrontar estos datos respecto de otras variedades.

\section{MATERIALES Y MÉTODOS}

\section{Materiales}

Las mazorcas de cacao (T. cacao) de la variedad criolla utilizadas como material de ensayo, fueron obtenidas en la vereda Totare, ubicada en el municipio de Alvarado, Tolima. Dentro de las condiciones geo-climáticas, tenemos las siguientes: altitud de la cabecera municipal (metros sobre el nivel del mar): 439 , clima templado; temperatura media: $26^{\circ} \mathrm{C}$, aunque hay zonas secas, posee 3 ríos de gran importancia como el Totaré, La China y Alvarado. Existe, además, un gran número de corrientes menores.

El procesamiento comprende cuatro etapas que serían el método de referencia para realizar los experimentos: la fermentación, el secado (a diferentes temperaturas y tiempos), la molienda y la extracción con solvente.

\section{Preparación de la muestra}

Las mazorcas de cacao se recogen, una vez maduras, se corta el pedúnculo leñoso. Para liberar las semillas, se abren las mazorcas de pared gruesa, golpeándolas con un simple mazo de madera. Las mazorcas, generalmente cambian de color al madurar, las verdes se colorean de amarillo oro, mientras que las mazorcas inmaduras rojas de otras variedades se colorean en amarillo naranja (Beckett, 1994), como se aprecia en la figura 1. 
Figura 1. Índice de madures para la mazorca de cacao utilizadas en el experimento, con el mismo grado de madurez, pero de variedades hibridas distintas, provenientes de la vereda Totare, municipio de Alvarado, Tolima.

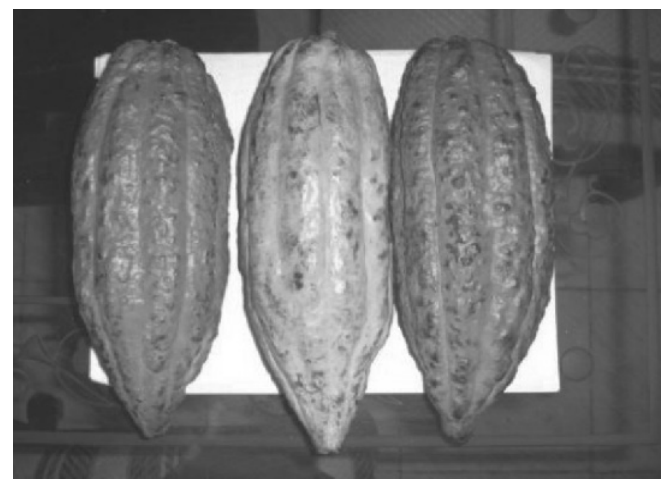

La fermentación se llevó a cabo en forma artesanal como lo realizan las comunidades indígenas, los campesinos y los cacaoteros de nuestra región, ya que en algunas partes del departamento del Tolima, principalmente en el Sur, a nivel económico, el cacao se comercializa ya fermentado y secado en fibras, por lo tanto, la fermentación se llevó a cabo de esta manera, en fibras como lo muestra la Figura dos; apilando una cantidad de granos frescos con la pulpa suficiente para que los microorganismos produzcan calor, elevando la temperatura a la vez que se permite un limitado acceso al aire entre almendras.

Figura 2. Fermentación de las habas de cacao provenientes de vereda Totare, municipio de Alvarado, Tolima.

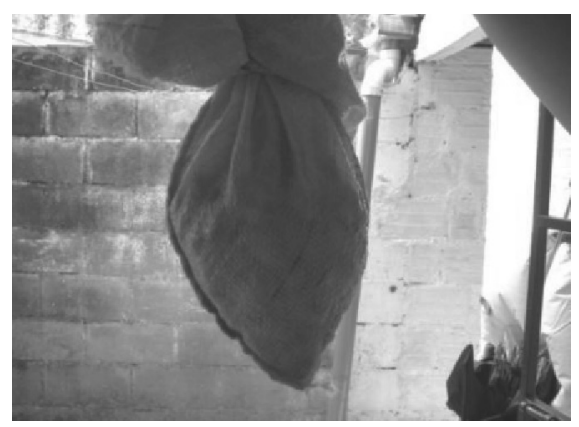

Luego, se separaron en cuatro muestras para someterse a diferentes tipos de secado. Posteriormente, los granos fueron molidos con un molino tipo casero. Por último, se hizo una lixiviación utilizando como solvente Éter-Etílico.

Para la determinación de contenido de humedad, acidez, $\mathrm{pH}$ y grasa, las muestras fueron llevadas al laboratorio bromatológico del hospital Federico Lleras Acosta de Ibagué. 


\section{Método experimental}

Dentro de las consideraciones a tener en cuenta al momento del inicio de la observación y la implementación del diseño experimental surgen los interrogantes; ¿Qué efecto tienen la composición del grano o almendra y la temperatura y tiempo en el proceso de extracción de la manteca?

Las semillas luego de la fermentación, fueron separadas en cuatro muestras aproximadamente de 176 g cada una.

a) La muestra número 1 fue sometida a un secado a temperatura ambiente durante 12 horas.

b) La muestra número dos (2) fue sometida a un secado a temperatura ambiente durante 24 horas.

c) La muestra número tres (3) fue sometida a un secado a una temperatura de $45^{\circ} \mathrm{C}$ durante 12 horas

d) La muestra número cuatro (4) fue sometida a un secado a una temperatura de $45^{\circ} \mathrm{C}$ durante 24 horas.

e) La muestra 5 son las semillas de cacao frescas trituradas.

\section{Determinación del contenido de humedad}

La determinación del porcentaje de humedad se hizo en un horno de circulación forzada de aire caliente Brinder. El contenido de humedad fue determinado midiendo la pérdida en la relación peso-masa a $105^{\circ} \mathrm{C}$ durante dos (2) h.

Determinación de acidez

El contenido de acidez se calculó por el método de potencial oxido-reductor, preparando una solución de Éter-Alcohol en proporción tres a uno (3:1); debido a que la muestra a analizar tenía un alto contenido de grasa o manteca, además de esto se utilizó una solución de $\mathrm{NaOH}$ $0.0092 \mathrm{~N}$.

Determinación del $\mathrm{pH}$

El pH fue establecido con un potenciómetro Orion Research modelo 601A y con un agitador Thermolyne 1.

\section{Determinación del contenido de grasa}

¿Qué relación tiene el tiempo y la temperatura a manera de proporción respecto de una eficiencia operacional que será la que más cantidad de manteca de cacao obtenga a partir del control de dichas variables?, ¿Son estas variables las que inciden a su vez directamente en el proceso de extracción sabiendo que son variables implícitas en el proceso de fermentación anterior a la operación de extracción? 
Para determinar el porcentaje de grasa o manteca, inicialmente se pesaron las muestras trituradas, - teniendo en cuenta que para el trabajo en laboratorio se tomó una muestreo al azar de la variedades utilizadas, y se realizó un trabajo experimental con una muestra representativa, - se depositaron en un beaker de $500 \mathrm{ml}$, luego se tomaron $250 \mathrm{ml}$ de solvente y se agregaron a las muestras 1, 2 y 3, a la muestra número 4 se le agregó sólo $150 \mathrm{ml}$ por tener un peso menor que las demás. Posteriormente se mezcló cada muestra con su respectivo solvente y se selló el paso del aire con ayuda de un fragmento de látex y una caja de petri, para evitar la volatilización del éter. Las muestras fueron agitadas cada 15 minutos, proceso que duró aproximadamente 2 horas. Luego se procedió a filtrar, (figura 3) para obtener la mezcla de éter-grasa; obtenida ésta se procedió a evaporar el éter a $150{ }^{\circ} \mathrm{C}$ en una plancha de calentamiento Lab-Line y en vasos de grasa previamente desecados.

El porcentaje de grasa o manteca se determinó por la diferencia de peso entre el vaso vacío y el vaso con el material graso.

Figura 3. Filtrado de la mezcla Éter-grasa realizada en el laboratorio Bromatológico del Hospital Federico Lleras Acosta E.S.E., municipio de Ibagué, Tolima.

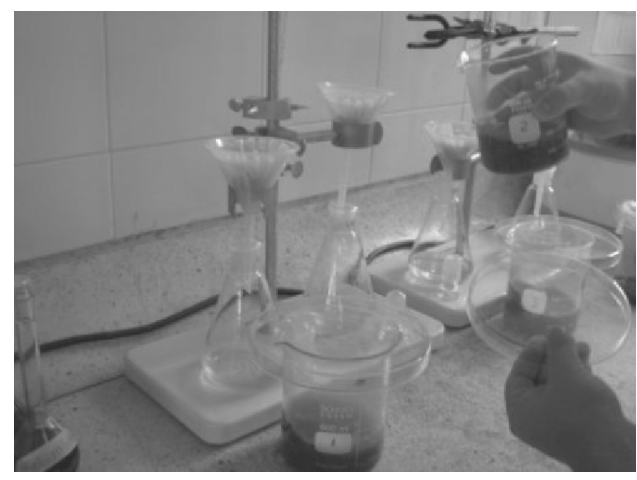

\section{RESULTADOS}

Todos los resultados expuestos en esta parte están calculados en base húmeda, ya que se supone que la materia seca es aquella a la que se le ha retirado humedad y la manteca. De acuerdo con la estadística descriptiva de tipo cuantitativo utilizada en el experimento, y con un nivel de confianza del 95\%; el porcentaje de humedad para la muestras uno (1), dos (2), tres (3) y cuatro (4) fue de 9.85, 8.94, 9.64 y $7.71 \%$ respectivamente, como se muestra a continuación en la figura 4., y se encontró que las muestras que se secaron durante 24 h, fueron las que menos porcentaje de humedad mostraron.

Los valores de $\mathrm{pH}$ fueron similares para todas las muestras como se aprecia en la figura 5, indicando que las habas de cacao trituradas, después de la fermentación y secado tienden a ser básicas. 
No se obtuvieron valores significativos de acidez expresado como ácido oleico, ya que se encontraban entre los rangos de $0.001 \%$ - $0.003 \%$ para todas las muestras incluyendo la muestra fresca. Esto, indicando que el producto presenta uno grado de acidez muy bajo.

En cuanto a la cantidad de grasa o manteca obtenida para $100 \mathrm{~g}$ de muestra se obtuvieron los siguientes resultados mostrados en la figura 6; que dependieron de las temperaturas y tiempos evaluados. La muestra 4 presentó el mayor porcentaje de manteca $27.3 \%$, seguida de la muestra 2 con $24.3 \%$, que fueron las que menor contenido de humedad presentaron, el contenido de humedad, por lo tanto, influye directamente sobre la cantidad de manteca interna en la almendra.

Las cantidades de grasa o manteca obtenidas presentaron características organolépticas típicas de ellas como su olor y sabor a chocolate. Por lo cual, comprobamos por qué es esta una de las razones de su utilización en la industria alimentaria, y por qué el cacao del departamento es de gran interés para la industria colombiana. La muestra número 5, es decir, las semillas de cacao frescas, mostró un $\mathrm{pH}$ de 3.9 , la humedad fue de $54.25 \%$.

\section{DISCUSIÓN Y CONCLUSIONES}

En todas las muestras no se logró determinar la acidez por titulación porque al agregar la fenolftaleína se tornó violeta inmediatamente y no se podía diferenciar al momento de utilizar la solución de $\mathrm{NaOH}$. Sin embargo, ya que no se obtuvieron valores significativos de acidez expresados como ácido oleico $(0.001 \%$ - 0.003\%) para todas las muestras incluyendo la muestra fresca, se puede deducir que el producto presenta un grado de acidez muy bajo, por esta razón no se podía realizar la acidez por titulación indicada. Al realizar el método de potencial oxido-reducción se utilizó una cantidad muy pequeña de la solución debido a que bajó muy rápido el valor potencial, por lo tanto, se podría concluir que el método del potencial oxido reductor y la cantidad utilizada para la determinación cuantitativa son inversamente proporcionales. A su vez, de acuerdo con los resultados, la muestra que tuvo el menor porcentaje de humedad, fue la que mayor porcentaje de grasa obtuvo; y fue la que se expuso a mayor temperatura y tiempo de secado; es decir, es el mejor método para obtener mayores cantidades de manteca. Así, este resultado marca un precedente importante para el trabajo a escala de laboratorio y a las posibles variables a tener en cuenta a nivel Piloto, para la extracción de grasa o manteca de cacao.

Al determinar los porcentajes de grasa o manteca en las cuatro muestras se encontró que la temperatura y el tiempo influyen sobre esta en forma directa, a partir de los resultados cuantitativos y cualitativos obtenidos.

Los cambios en el pH después de la fermentación aumentan volviendo más básico el producto y, de paso, disminuyendo la acidez. Estos valores son consecuentes con aquellos encontrados por otros autores sobre los cambios ocasionados durante la fermentación de los granos de cacao. 


\section{REFERENCIAS BIBLIOGRÁFICAS}

BECKETT, S.T. (1994), Fabricación y utilización industrial del chocolate. Ed. Acribia, S.A., Zaragoza España.

BERNARDINI, E. (1981), Tecnología de aceites y grasas. Ed. Alhambra. Madrid, España.

CADENA DEL CACAO. (2009), Disponible en línea: www.agrocadenas.gov.co extraído el día 5 octubre.

PACHECO LÓPEZ, Dency José; TABORDA MARTÍNEZ, Manuel Enrique; DE LA ROSA, Catalino. Estudio fitoquímico preliminar y evaluación de la actividad antifúngica de los extractos de hojas, cortezas y semillas de Thevetia peruviana (Persoon) Schum Extraido el 29 de octubre de 2009 de la pagina Web: http://editorial.unimagdalena.edu.co/revistas/ index.php/intropica/article/view/121

RIVERA, Edgard y ALVIS, Carlos. Estudio analítico de las semillas de cacao (Theobroma cacao). Universidad del Tolima.

TSCHEUSCHNER, Horts-dieter. Fundamentos de tecnología de los alimentos. Ed. Acribia S.A., Zaragoza, España. 2001, pág. 23-25 